\title{
Mini-commentary on BJOG-20-1978.R1. Complement Receptors in Preeclampsia: Cleaning up Placental Debris
}

\author{
Richard Burwick ${ }^{1}$ \\ ${ }^{1}$ Cedars-Sinai Medical Center
}

January 28,2021

Mini Commentary: BJOG-20-1978.R1

Title: Complement Receptors in Preeclampsia: Cleaning up Placental Debris

Author: Richard M. Burwick, MD, MPH

Affiliation: Department of Obstetrics and Gynecology, Cedars-Sinai Medical Center, Los Angeles, CA

Text:

Complement proteins are central to the innate immune system and serve a critical role in host defense against foreign pathogens. However, complement proteins are also critical for normal clearance of damaged and apoptotic host cells, through the process of opsonization. This process is particularly critical in pregnancy, due to the large burden of semi-allogenic placental apoptotic debris released into the maternal circulation throughout gestation (Huppertz et al. Placenta 2003; 24:181-190). In placentally mediated disorders such as preeclampsia, there is an increase in apoptotic and necrotic placental debris, leading to an excess inflammatory response (Redman et al. Placenta 2000; 21:597-602). To achieve normal pregnancy, placental apoptotic debris must be cleared in an orderly fashion.

Complement component $\mathrm{C} 3 \mathrm{~b}$, the central opsonin in the complement cascade, marks apoptotic cells for phagocytosis. Accumulation of C3b leads to activation of the terminal complement pathway, which propagates inflammation and cellular injury, while cleavage of C3b (by complement factor I) leads to generation of iC3b and reduced complement activation. Generation of $\mathrm{iC} 3 \mathrm{~b}$ is important because complement receptors on phagocytes recognize iC3b, facilitating clearance of apoptotic cells. Complement receptors, and the interaction between complement receptors and their ligands, are therefore critical to complement homeostasis in normal pregnancy.

In this journal of BJOG, Lokki et al. sought to determine if genetic variants in complement receptors predispose women to develop preeclampsia. Investigators performed targeted exome sequencing of 11 genes coding for complement receptors, in 609 preeclampsia cases and 2,092 controls from two cohorts (The Finnish Genetics of Preeclampsia Consortium; National FINRISK study cohort). They discovered that missense variants in genes coding for complement receptor 3 (CR3) and complement receptor 4 (CR4), which are $\beta 2$-integrins that bind iC3b and are widely expressed on phagocytic cells, were significantly associated with preeclampsia. Through functional studies, Lokki et al. showed that two missense variants caused increased binding of CR3 and CR4 to iC3b, respectively, while another variant led to decreased binding of CR4 to iC3b. Other variants in complement receptors, including C3aR, C5aR, C1qR, CR1 and CR2, were not significant or of borderline significance.

Notably, missense variants in CR3 and CR4 were common, with minor allele frequency $11-15 \%$ among controls compared with 15-20\% among preeclampsia cases. Thus, while these variants are not causative of preeclampsia in isolation, they may predispose to preeclampsia in combination with other risk factors. While 
it is presumed that these variants in CR3 and CR4 lead to pro-inflammatory effects as seen in preeclampsia, this was not investigated in the current study. Thus, further studies are needed to determine if altered binding of CR3 and CR4 to iC3b, as well as other ligands, impacts cytokine release and inflammatory effects in preeclampsia. Regardless, the current study is an important addition to the rapidly expanding literature showing that complement dysregulation predisposes to preeclampsia. With the report that complement blockade may be an effective strategy to treat preeclampsia (Burwick et al. Placenta 2013; 34:201-3), there is an urgent need to identify women who may be predisposed to altered complement regulation in pregnancy.

Disclosure: RMB has received speaker fees and research grants from Alexion Pharmaceuticals

\section{Contact information:}

Richard M. Burwick, MD, MPH

Cedars-Sinai Medical Center

8635 W. $3^{\text {rd }}$ St., MOT, Suite $160 \mathrm{~W}$

Los Angeles, CA 90048

Mobile: 310-781-0230

Office: 310-423-6454; Mobile: 310-781-0230

Fax: 310-423-0140

E-mail: richard.burwick@cshs.org 